Revista Científica General José María Córdova, Bogotá, Colombia, julio-diciembre, 2017

DERECHOS HUMANOS Y DERECHO INTERNACIONAL HUMANITARIO

Vol. 15, Núm. 20 pp. 85-100 ISSN 1900-6586

Cómo citar este artículo: Hurtado Granada, M. I. (2017, julio-diciembre). Los límites del DIH a las armas autónomas. Rev. Cient. Gen. José María Córdova, 15(20), 85-100. DOI: http://dx.doi.org/10.21830/19006586.176

\title{
Los límites del DIH a las armas autónomas*
}

DOI: http://dx.doi.org/10.21830/19006586.176

Recibido: 1 de marzo de 2016 • Aceptado: 5 de junio de 2017

The Strictures of IHL Against Autonomous Weapons

Des limites du DIH aux armes autonomes

Os limites do DHH para as armas autônomas

Martha Isabel Hurtado Granada ${ }^{a}$

* Producto resultado del proyecto de investigación titulado "Inteligencia Tecnológica", del Grupo Investigación CIGA.

a Escuela de Inteligencia y Contrainteligencia "BG. Ricardo Charry Solano”, Bogotá, Colombia. Magíster en Cooperación al Desarrollo de la Universidad Autónoma de Barcelona y DEA en Derecho Internacional Público de la Universidad Autónoma de Barcelona. <Martha.martty77@gmail.com>. 
Resumen. Las armas autónomas tienen por finalidad identificar y atacar objetivos de manera independiente sin intervención humana. Por ahora, dichos sistemas se encuentran en posiciones fijas y funcionan por cortos periodos y contra objetivos limitados, pero en el futuro podrían ser operados por fuera de estos límites. Es evidente que el Derecho Internacional Humanitario (DIH) no tiene norma positiva que limite el uso de estas armas y que las nuevas tecnologías ponen en discusión quién libra la guerra, ¿las máquinas o el ser humano? En las leyes que rigen la guerra, las nuevas tecnologías no se encuentran expresamente prohibidas ni reguladas en los límites que los principios del DIH determinan. Por lo anterior, distinguir entre combatientes y no combatientes, el hecho de preservar la vida de los no combatientes o de los que han dejado de combatir estaría, eventualmente, determinado por una máquina. Asimismo, el DIH da preferencia a las armas que realizan ataques más precisos y que por lo tanto reducen la pérdida incidental de vidas humanas y sufrimiento innecesario. Pese a que las ventajas de dichas armas dependerán de su contexto, el debate consiste en determinar hasta qué punto sus efectos se encuentran enmarcados en los principios del DIH.

Palabras clave: armas autónomas; Derecho Internacional Humanitario; ética; límites al DIH; principios humanitarios.

Abstract. The Autonomous weapons are intended to identify and attack targets independently without human intervention. For now, such systems are in fixed positions and work for short periods and with limited objectives, but in the future these weapons could be operated outside these limits. Clearly, the Humanitarian Law (IHL) has not positive rule that limits the use of these weapons and new technologies call into question who wages war, machines or humans? In the laws governing war new technologies they are not expressly prohibited or regulated within the limits determined IHL principles. Therefore, distinguish between combatants and noncombatants, the fact preserves the lives of noncombatants or those who have stopped fighting would be possibly determined by a machine. Moreover, International Humanitarian Law gives preference to weapons so make more precise attacks to reduce incidental loss of life and unnecessary suffering. However, the advantages of such weapons depends on its context, the debate is to determine to what extent the effects of these weapons are framed on the principles of IHL.

Keywords: autonomous weapons; ethics; humanitarian principles; International Humanitarian Law; strictures of IHL.

Résumé. Les armes autonomes sont destinées à identifier des cibles d'attaque de façon indépendante, sans intervention humaine. Pour l'instant, ces systèmes sont dans des positions fixes et de travail pour de courtes périodes et avec des objectifs limités, mais à l'avenir ces armes pourraient être utilisées en dehors de ces limites. De toute évidence, le droit humanitaire (DIH), le droit international n'a pas de règle positive qui limite l'utilisation de ces armes et de nouvelles technologies remettent en question qui guerre des salaires, des machines ou des humains ? Dans les lois qui régissent les nouvelles technologies de guerre, ils ne sont pas expressément interdits ou réglementées dans les limites fixées principes du droit international humanitaire. Par conséquent la distinction entre combattants et non-combattants, le fait de préserver la vie des non-combattants ou ceux qui ont cessé de se battre serait probablement déterminée par une machine. En outre, le droit international humanitaire accorde la préférence aux armes pour faire des attaques plus précises pour réduire les pertes en vies humaines et des souffrances inutiles. Cependant, les avantages de ces armes dépendent de son contexte, le débat est de déterminer dans quelle mesure les effets de ces armes sont encadrés sur les principes du droit international humanitaire.

Mots-clés: armes autonomes; droit international humanitaire; l'éthique; limites aux DIH; principes humanitaires. 
Resumo. As armas autônomas, visam identificar e atacar alvos forma independente, sem intervenção humana. Por enquanto, esses sistemas estão em posições fixas e trabalham por períodos curtos e com objetivos limitados, mas no futuro essas armas poderão ser operadas fora destes limites. Claramente, o Direito Humanitário (DIH), o direito internacional não tem nenhuma regra positiva que limita o uso dessas armas e novas tecnologias póem em discussão quem livra a guerra, máquinas ou seres humanos? Nas leis que regem as guerras essas novas tecnologias não são expressamente proibidas ou regulamentadas dentro dos limites determinados princípios do Direito Internacional Humanitário. Portanto distinguir entre combatentes e náo combatentes, o fato de preservar a vida dos náo-combatentes, ou daqueles que pararam de lutar, seria possivelmente determinada por uma máquina. Por outro lado, o direito internacional humanitário dá preferência a armas capazes de fazer ataques mais precisos para reduzir a perda acidental de vida e sofrimento desnecessário. No entanto, as vantagens de tais armas dependem de seu contexto, o debate consiste em determinar até que ponto os efeitos dessas armas estáo enquadrados nos princípios do DIH.

Palavras chave: armas autônomas; direito internacional humanitário; ética; limites doDIH; princípios humanitários.

\section{Introducción}

Los avances tecnológicos siempre han sido empleados por los ejércitos en aras de lograr una mayor ventaja contra su enemigo. Las armas autónomas no iban a ser la excepción. Las nuevas tecnologías que forman parte de la cuarta revolución industrial (Khun, 1970) están siendo utilizadas en diversos rincones de la Tierra con la finalidad de debilitar el actuar del enemigo, obtener información en tiempo real del enemigo y conseguir mayores resultados en el campo de batalla.

Este trabajo parte de la siguiente pregunta: ¿es necesario poner límites a las nuevas armas autónomas? A priori se puede afirmar que todo armamento debe ser examinado, según lo estipulado por el Artículo 36 del I Protocolo Adicional de 1977, con la finalidad de revisar si cumple con dos principios básicos del Derecho Internacional Humanitario (DIH): que dicho armamento no actúe por sí mismo de manera indiscriminada y que no cause daños superfluos y sufrimientos innecesarios.

Por lo anterior, este trabajo tiene por objetivo realizar un estudio analítico de las normas del DIH existentes que eventualmente limitan las armas autónomas. Específicamente, se hace una revisión de normas aplicables a los conflictos armados internacionales, tales como los principios y el I Protocolo Adicional de 1977.

Para cumplir el objetivo se consultaron fuentes primarias de información que brindan respuestas académicas cualitativas a la pregunta problema. En dicha búsqueda, se observó que el debate académico se centra en si es necesario pactar o no límites a las armas autónomas, lo cual permitió el vacío jurídico para limitar el nuevo armamento. Por esta razón, lo más sólido, en materia de protección, son los principios del DIH por cuanto imponen límites a los modos, lo cual representa un nuevo desafío a los juristas a nivel mundial.

Aunque el debate epicentro de este documento tiene un gran contenido ético y moral, el análisis solo se centra en los aspectos jurídicos que limitan los medios para hacer la guerra. 


\section{Los desafíos del DIH}

La constante evolución de la guerra genera el afán por clasificar todas las expresiones de las hostilidades, las cuales responden a una categorización analítica que describe las causas, los actores, las técnicas y las tácticas de los conflictos que surgen en el ámbito global (Mejía \& Chaíb, 2012).

El Comité Internacional de la Cruz Roja (CICR), como guardián del DIH, determina que el mayor desafío al que se enfrenta el DIH es la capacidad del conjunto normativo para proteger a los civiles. La complejidad de los conflictos armados actuales ha puesto en debate tanto la propia clasificación que hace el DIH de estos, como los modos actuales con los cuales se llevan a cabo las hostilidades.

Aunque suene contradictorio, el DIH busca humanizar algo que por sí es brutal y cruel. No pretende justificar la guerra, solo se propone moderar el comportamiento de los combatientes, en aras de poder restablecer los Estados tras las hostilidades. Cuando en un conflicto se ha transgredido constantemente las normas del DIH, al pactar la paz entre las partes se dificulta que esta sea permanente y generalizada (Kalshoven \& Zegveld, 2005).

Respecto a los innumerables desafíos que el DIH enfrenta en la actualidad, en este documento se hará referencia únicamente a los modos de hacer la guerra, es decir, a la normatividad compilada en el derecho de la Haya que regula los aparatos, dispositivos y armas para hacer la guerra, en especial lo concerniente a la nueva tecnología, que representa un desafío para el DIH.

Los modos de hacer la guerra siempre han brindado un punto importante a la eficacia del $\mathrm{DIH}$, lo que no suele suceder con los medios para hacer la guerra. Ello se debe a que las tácticas y las doctrinas militares suelen estar ajustadas al deber ser de la norma humanitaria, mientras que los avances tecnológicos superan al quehacer doctrinal y legal, al tiempo que generan debates políticos y económicos que, en la mayoría de las ocasiones, enlodan la protección de las víctimas en los conflictos y la supremacía de los principios del DIH.

La época actual no puede ser la excepción y las armas autónomas son un desafío jurídico cuando tienen por objetivo identificar y atacar objetivos de manera independiente, sin intervención humana. Por ahora, dichos sistemas se encuentran en posiciones fijas y funcionan de manera autónoma por cortos periodos y contra objetivos limitados, pero el futuro de estas armas está dispuesto para que sean operadas por fuera de estos límites. Es evidente que el DIH no tiene norma positiva que limite el uso de estas armas y que las nuevas tecnologías ponen en discusión quién libra la guerra, ¿las máquinas o el ser humano?

En las leyes que rigen la guerra, las nuevas tecnologías no se encuentran expresamente prohibidas, aún peor, no están reguladas y no se ha determinado si su actuar se ajusta a los límites que los principios del DIH estipulan. Por lo anterior, esta tecnología representa un reto jurídico, pues eventualmente sería una máquina la encargada de distinguir entre combatientes y no combatientes, así como de preservar la vida de los no combatientes o de los que han dejado de combatir (International Comitee of the Red Cross [ICRC], 2012). Asimismo, el DIH da preferencia a las armas que realizan ataques más precisos y por lo tanto reducen la pérdida incidental de vidas humanas y sufrimiento innecesario. Pese a que las ventajas de dichas armas dependerán de su contexto, el debate consiste en determinar hasta qué punto los efectos de estas armas se encuentran enmarcados en los principios del DIH. 


\section{De las armas autónomas}

En lo referente al objetivo de este trabajo, se hace necesario comprender qué significa arma o medio para hacer la guerra. En ese orden de ideas, para las Fuerzas Militares de Colombia, Ejército Nacional, las armas son los medios que el Estado suministra a sus Fuerzas Armadas para que puedan usar la fuerza en conflicto armado (Ejército Nacional, 2010). Dicho concepto que trae el Reglamento militar se encuentra inmerso en los límites que el DIH determina para el uso de los medios, los cuales son mencionados taxativamente. Igualmente, se encuentran enmarcados en aquellos límites que definen el honor y la necesidad militar. Finalmente, la norma hace el esfuerzo por clasificar las armas según su índole.

No obstante, hasta el momento las armas autónomas no se encuentran clasificadas ni delimitadas por el DIH ni por los manuales de operaciones militares que conforman la doctrina del actuar en la guerra. Lo anterior implica que su uso aún no es masivo ni constante, pero es importante observar cómo la tecnología informática actualmente impulsa un rápido incremento en las capacidades autómatas de los robots, que se emplean tanto en la vida doméstica como en el armamento militar, siendo los Drones los que captan la mayor atención (Horowitz \& Scharre, 2015).

Ciertamente, este tipo de tecnología ha representado un avance para las fuerzas militares del mundo entero, toda vez que permite al combatiente estar ausente en las zonas de operaciones militares, lo cual reduce el número de bajas de combatientes y reduce su propio riesgo. Igualmente, son armas más precisas que detectan sus blancos con mejor resultado y por lo tanto disminuyen los dańos colaterales propios de las hostilidades, pues permiten ubicar al enemigo o a la amenaza de manera más clara.

No obstante, el CICR ha aseverado que la distancia entre el operador y su adversario implica una desconexión emocional, lo cual hace más probable que las violaciones al DIH sean más recurrentes. El organismo también pone en duda la capacidad del operador para procesar en tiempo real la gran cantidad de datos que este tipo de máquinas obtienen en corto tiempo y, por ende, cuestiona el cumplimiento de las normas del DIH cuando se pone en curso una operación (The Red Cross and Red Crescent, 2011).

La inteligencia artificial es una característica de este tipo de armas, lo cual supone que están preparadas para dar respuesta ante los cambios del terreno y de las condiciones que se presentan, de modo que, en este supuesto, las armas autónomas tienen la capacidad de adaptarse según el entorno y sus cambiantes condiciones hostiles. La duda se presenta cuando estos sistemas deben distinguir entre un objetivo civil y otro militar de manera autónoma, y también cuando se reflexiona si su decisión cumple con los principios del DIH. Tal como lo ha mencionado el CICR, el despliegue de tales sistemas reflejarían un cambio de paradigma y un cambio cualitativo importante en la conducción de las hostilidades.

Por su parte, los Estados están empezando a cuestionarse sobre los desafíos y las oportunidades relacionados con el aumento de la capacidad de autonomía en las armas. Incluso, son pocos los Estados que tienen previsto el avance tecnológico y la forma en que deben ser incluidos en sus futuros sistemas de armas, lo cual es muestra del riesgo que se está corriendo a nivel mundial al adquirir este tipo de armamento (United States, 2012). 
Esto se debe a que un arma de este tipo implicaría un gran despliegue tecnológico y enormes esfuerzos de programación, pues supondría que tendría la capacidad de distinguir y discernir en múltiples circunstancias que suponen el uso de la razón humana; por ejemplo, distinguir a un combatiente armado de un campesino con una escopeta propia para la caza. Igualmente, supone que el arma sería capaz de realizar o diseñar estrategias que implican ventaja militar o emitir juicios relativos a la proporcionalidad militar en diversas situaciones, incluso en ambientes cambiantes (The Red Cross and Red Crescent, 2011).

En el caso de los Aviones No Tripulados (UAV, por su sigla en inglés), es importante resaltar que, a pesar de que son un gran componente militar de Estados Unidos, hasta el momento no están preparadas las máquinas para disparar autónomamente (Zenko, 2013). Estas máquinas, por ahora, requieren de pilotos en tierra que los controlen, pero su desarrollo es cada vez mayor y se están automatizando más: por ejemplo, en funciones como aterrizaje o ciertos vuelos, incluso hoy en día un solo piloto logra controlar varios UAV al mismo tiempo. Por lo anterior, se estima que los aviones caza, en un futuro, no serán pilotados sino controlados y su aspecto automatizado implicará mayor reacción en vuelo, así como impredictibilidad de las tácticas aéreas (Anderson \& Waxman, 2013).

Por los avances de la inteligencia artificial, es importante observar la genuina autonomía de las máquinas y su capacidad de adaptación al entorno, igual o mejor que la del ser humano, pero reduciendo el riesgo de pérdidas humanas. Este hecho hace que sea determinante estudiar su aplicación en la guerra. Inicialmente, estos sistemas están diseńados para ser empleados en entornos operativos ética y legalmente menos problemáticos (Anderson \& Waxman, 2013). Es importante resaltar que hasta el momento nadie espera que las máquinas reemplacen, totalmente, a los combatientes en las hostilidades, es decir, se hace necesaria la presencia del estratega en el campo de batalla, sus decisiones y su responsabilidad en la actuación.

Ahora bien, ¿qué sucede en el caso que por el uso de un arma autónoma se produzca un crimen de guerra o cualquier conducta reprochable por el derecho?, ¿quién es el culpable?, ¡¡l programador, el fabricante, el operador o el comandante? El DIH no debe cambiar cada vez que los avances tecnológicos demanden una regulación, es la tecnología la que debe ser realizada acorde con los principios básicos del DIH, siempre pensando en lo primordial: proteger al no combatiente y al que ha dejado de combatir.

De la misma forma que lo afirman Anderson \& Waxman (2013), las armas autónomas representan un doble desafío, uno ético y otro legal. El ético consiste en la capacidad de la máquina para responder ante sus actos y el legal en su responsabilidad frente a las normas que deben regular su aplicación y empleo.

\section{De la regulación de los desafíos al DIH}

Las discusiones que han surgido en el mundo sobre la fabricación de nuevo armamento de alto nivel tecnológico recuerdan las que se plantearon cuando se crearon elementos como los submarinos y la aviación. Discusión que tenía un alto componente del honor militar, pues atacar desde una posición segura y confortable no se consideraba como honorable, de hecho llegaba a ser 
deshonroso. No obstante, en su tiempo fue indispensable escuchar en el debate las ventajas que representaba para los ejércitos el empleo de estos vehículos.

Es así como en la Convención de 2014 de Naciones Unidas, la discusión sobre este tipo de armamento se centró sobre el control humano (The Convention on Certain Conventional Weapons [CCM], 2014). Muchos participantes decidieron apoyar la idea de prohibir el armamento completamente autónomo y plantearon como requisito para operar dichas armas el control humano, pues consideran que el ser humano tendrá la capacidad de responder ante los yerros que una mala decisión puede acarrear en medio de las hostilidades. No obstante, han dejado de lado un tema relevante y es la precisión de este tipo de armamento y las ventajas militares que representa, lo cual son avances tecnológicos que difícilmente se podrán detener. Asimismo, el Meeting de abril de 2015 se centró en tratar de comprender el funcionamiento de los sistemas autónomos, sin duda un avance para que el campo jurídico entienda a la ingeniería, en aras de la construcción de leyes efectivas y aplicables (CCM, 2015).

Por su parte, el DIH tiene su origen tanto en el derecho de Ginebra, como en el derecho de la Haya. El primero pretende proteger a los no combatientes o a quienes han dejado de combatir, y el segundo regula los aspectos referentes a la conducción de las hostilidades. Ambas fuentes han ido evolucionando con la finalidad de restringir el uso de la fuerza sin medida, siendo particular que el fin último del derecho de la Haya es limitar los métodos y medios para hacer la guerra (Bugnion, 2011).

En realidad, la línea divisoria entre ambas fuentes es muy delgada y, por lo tanto, hay normas que pertenecen a una y otra. Al inicio, las dos ramas se podían identificar por sus fines, pero al buscar un punto en común (humanizar la guerra), fue necesaria unirlas y se presentó con los Protocolos Adicionales de 1977, los cuales buscaron exaltar los principios, no su origen, aplicables a las dos tipologías de guerra hasta entonces conocidas.

Cabe añadir, de modo anecdótico, que desde los Convenios del 49 el CICR (1986) ha sido el custodio del Derecho de Ginebra, mas no fue así para los temas relativos al derecho de la Haya. Los Estados no reconocían plenamente al CICR para que se encargara de los asuntos propios del derecho de la Haya, en especial en el tema del desarrollo tecnológico. Por ello fue necesario que se llevaran a cabo varias Conferencias Internacionales del Movimiento para que el Comité tuviese esa facultad.

Finalmente, la conducción de las hostilidades con frecuencia tiene dificultades cuando se revisa su regulación, toda vez que puede o no estar frente a una violación del derecho o puede estar frente a un vacío normativo. En algunos casos, como las bombas atómicas o las minas antipersonas, los modos de hacer la guerra violaban los principios del DIH per se. En otros casos, por el contrario, hay que tener en cuenta varios factores para determinar su violación, como suele suceder al valorar los métodos para hacer la guerra (Bugnion, 2011).

Respecto a la limitación de los medios y métodos, ha sido necesario considerar elementos que salen de lo humanitario para entrar en temas propios de la defensa y la seguridad de los Estados, en aras de proteger a los civiles y evitar la proliferación de los Conflictos Armados no Internacionales (Cani).

Es así, y a modo de ejemplo, la Declaración de San Petersburgo (1868) dio el primer paso en la prohibición de armamento. Para esa época, se inventaron unos proyectiles cuya finalidad era 
destruir los vagones de municiones, pero cuando impactaban en un ser humano causaban mucho sufrimiento. El zar de Rusia, quien convocó una Comisión Internacional para dicho propósito, argumentó que, si bien es legítimo debilitar al enemigo, dicho armamento "agravaría inútilmente los sufrimientos de los hombres puestos fuera de combate" (Doménech, 2007, p. 314). Tal como se observa, la importancia de la declaración radica en los principios que de ella se desprenden, más que en la propia prohibición, pues se trata de prohibir un artefacto que causa sufrimiento innecesario al tratar de debilitar al enemigo.

Igualmente, y también a modo de ejemplo, se pueden ver los resultados de la Conferencia Internacional de la Paz, cuya propuesta, además del mantenimiento de la paz, era codificar las costumbres y las leyes de la guerra terrestre, que tuvo inspiración en el código de Lieber (1863). De ello se estableció un reglamento (1899-1907), cuyo Artículo 22 conserva el principio más importante: "Los beligerantes no tienen un derecho ilimitado en cuanto a la elección de medios para dañar al enemigo" (Convención II de la Haya, 1899). Del anterior artículo se puede observar que existen límites para los combatientes y que no todo es válido en la guerra. Asimismo, de aquí se desprende la importante prohibición consagrada en el Artículo 23 que propende por evitar un daño superfluo. De acuerdo con Doménech (2007), superfluo implica el empleo de un arma que no justifica el sufrimiento causado y que su utilidad es mínima o inexistente, de manera que desequilibra la balanza hacia el primer aspecto.

Por lo anterior es que se contempla, en el ámbito normativo, que toda creación de nuevo armamento debe ser valorado desde conceptos políticos y éticos. Sin embargo, también es cierto que esta valoración encierra uno de los debates más críticos en la política: la capacidad para desarrollar nuevo armamento que permita una ventaja en el campo de batalla para defender los intereses nacionales y la necesidad militar, versus la protección debida a los civiles y la prohibición de causar daños superfluos y sufrimiento innecesario.

\section{El desafío desde el ius gentium del DIH}

Desde la época de Suárez (s. XV) ha existido la pregunta de si el derecho de gentes es derecho humano positivo. En efecto, para Suárez el derecho de gentes es derecho humano positivo y consuetudinario internacional, el cual es anterior al consentimiento de los pueblos que conforman el derecho escrito convencional y, por tanto, surge de las prácticas de los pueblos en la comunidad internacional y como tal tiene su fundamento en la naturaleza del ser humano (Herrera, 2008).

Ahora bien, en oposición al positivismo jurídico se encuentra el iusnaturalismo. Es así como Hobbes realiza la distinción entre estado natural y el estado sociedad, a partir de la cual determina que en el estado natural existe la guerra y los intereses particulares. Sin embargo, en su concepto, el ser humano requiere de cierto orden que se caracteriza por el temor, lo cual conduce a que las normas sean necesariamente escritas. Por lo anterior, el hombre se somete a algo superior, a la norma. Pero cuando se trata de Estados, sobre estos no hay un superestado, por lo que viven en estado de naturaleza. Por ello, no puede existir un derecho positivo internacional, sino solo máximas de la razón (Verdoss, 1967). 
En este orden de ideas, el derecho natural resulta de la razón, mientras el derecho positivo resulta de la voluntad de un superior. Desafortunadamente, Hobbes tenía razón en un aspecto: la naturaleza, tal como lo muestra la historia, es que el ser humano y por supuesto los Estados viven en guerra. Debido a que no hay posibilidad de lograr el desarrollo humano sin orden, los Estados se ven en la necesidad de pactar unas reglas generales para lograr una coexistencia. Es así como nace el Derecho Humanitario, concebido como hoy en día lo conocemos.

Una de las características del Derecho Internacional contemporáneo es la humanización (Carrillo, 2001), siendo el DIH el principal exponente de dicho objetivo al procurar humanizar la guerra a través de la protección a quienes no combaten y a los límites pactados para los medios y métodos. Ahora bien, como fuentes secundarias de este derecho, los principios del derecho son establecidos como normas propias del derecho natural y se caracterizan por ser intemporales. En términos del DIH, los principios parten de la distinción entre quienes combaten y los que no, igualmente se fundamentan en la idea de la humanización de la guerra, para lo cual dichos principios se pueden encontrar pactados expresamente en las normas escritas (tratados, convenios y protocolos), así como encontrarse implícitamente o en la propia costumbre. Los principios tienen por finalidad brindar humanidad en el actuar de los combatientes y ser una guía en la interpretación normativa (Salmón, 2004).

Los principios del DIH que estructuran la función armada son tres: el combatiente, los objetivos militares y los medios (armas) y métodos (procedimientos). Tal como se ha mencionado anteriormente, el DIH basa su normatividad en la regulación de estos tres elementos mediante la formulación de unos principios: distinción, limitación y proporcionalidad (Doménech, 2007).

Ahora bien, en lo referente a la ratio personae, se puede determinar que la limitación hace referencia al principio por excelencia de la protección debida a los civiles (Pictet, 1998). De este principio general se derivan cinco más:

1. Se debe hacer distinción entre la población civil y el combatiente.

2. No pueden ser objeto de ataques ni represalias la población civil ni las personas civiles (art. 51, I Protocolo Adicional).

3. Quedan prohibidos los actos que causen terror en la población civil (art. 51, I Protocolo Adicional).

4. En todo caso se deben tomar todas las precauciones para salvar a la población civil y buscar reducir el impacto de las hostilidades en sus bienes e integridad física (art 57 y 58, I Protocolo Adicional).

5. Solo los miembros de la fuerza pública pueden atacar a su enemigo y resistirle.

Tal como se observa, existen principios que buscan proteger a las personas que no combaten o que han dejado de combatir. En ese orden de ideas, el aspecto relevante para el presente trabajo es la distinción entre combatientes y no combatientes. Respecto a las armas autónomas, estas deben estar programadas para que en su modo autónomo logren distinguir entre un combatiente herido o enfermo, uno que depone sus armas, un prisionero de guerra o un civil que no combate. Igualmente, tienen que tener la capacidad de diferenciar los bienes civiles, los bienes mixtos y los bienes militares. 
Otro de los principios es el ratione conditionis, que prohíbe las armas y métodos de guerra que causan pérdidas inútiles o sufrimientos excesivos. Este principio busca no solo proteger a las personas que no participan en las hostilidades, sino también evitar sufrimientos innecesarios a los combatientes (Pictet, 1998).

Lo anterior es la prueba de la insuficiencia de los derechos naturales, insuficiencia que ha inspirado a Locke a determinar que la positivización de los derechos debe ser un proceso asumido e incorporado por el poder porque la fundamentación racional no es suficiente (Segovia, 2014). Por esto es indispensable pensar las otras fuentes del derecho internacional: la costumbre y los principios, ¿son realmente fuentes del derecho internacional?

El derecho internacional, como el derecho interno, puede no contar, y generalmente no cuenta, con normas expresas para decidir casos concretos, pero la función de la jurisprudencia es resolver los conflictos de derechos e intereses contrapuestos aplicando, a falta de provisión jurídica específica, lo corolarios de los principios generales y encontrar - exactamente igual que las matemáticas - la solución del problema. Este es el método de la jurisprudencia y por el cual el derecho se ha desarrollado progresivamente en cada país, de tal manera que ha delimitado y solucionado las relaciones jurídicas tanto entre Estados como entre particulares (Tribunal Arbitral Reino Unido y Estados Unidos, 1923).

\section{El desafío desde el positivismo del DIH}

La característica del derecho positivo es que tiene una base sociológica y una base normativa. De la conexión de estas dos variables resulta que la norma presupone un valor que por sí misma debe realizarse. Contrario a ello se plasma la idea del positivismo dogmático, en el que no hay una norma suprapositiva, de manera que reduce todo a una norma positiva.

En aras de lo anterior, y tal como lo afirma Mauricio Plazas Vega (2006), desde hace muchos años el derecho positivo acude a la moral para establecer las bases y los efectos jurídicos de acciones particulares, sin que ello implique una negación o una pérdida de la dimensión externa de la ley y de los preceptos y principios de la moral.

Para Dunant (2010), tras los hechos de Solferino se hizo indispensable pactar un mínimo en la guerra, mínimo que fuese respetado por todos, tanto en tiempos de paz como en tiempos de guerra. Se trataba de la neutralidad del cuerpo sanitario y médico. Siendo este un concepto novedoso en el arte de la guerra, ha sido uno de los más perdurables. Este principio, concebido en los diez artículos del primer acuerdo del continente europeo ("Tratado de amistad y de paz" mencionado anteriormente), no es otra cosa que la necesidad de pactar por escrito la palabra de los grandes señores del Estado, lo cual demuestra que el derecho natural, cuando se habla de relaciones internacionales, no es evidente ni general, por lo que es necesario pactarlo, codificarlo y ratificarlo.

De algo sí está segura la comunidad internacional, lo que no está pactado por escrito y debidamente ratificado, no es vinculante. Es así como tras los crudos hechos de la Segunda Guerra Mundial surgieron los cuatro Convenios de Ginebra de 1949, suscritos y ratificados por todos 
los Estados hasta el momento existentes, siendo estos convenios las normas más universales que jamás se han escrito.

Tal como se ha podido observar, el Derecho Internacional se fundamenta principalmente en el consentimiento escrito de los Estados. Pero dichas normas no necesariamente dan respuesta a un problema o regulan alguna situación en particular, por ello la indagación consensualista no termina en el tratado o el convenio, sino que podría estar en el propio comportamiento de los Estados o mediante la aplicación de los principios generales del derecho.

Es así como, para hablar de Derecho Humanitario, se hace indispensable pensar en el conjunto normativo, escrito y no escrito, natural y positivo, que procura limitar los medios y métodos de la guerra, así como proteger a las personas, especialmente a los que no participan de ella (Rodríguez-Villasante \& Prieto, 2007).

A pesar de la cantidad de normas existentes no escritas, al finalizar la Segunda Guerra Mundial se hizo necesario que la comunidad internacional se pusiera de acuerdo en los mínimos en las guerras o Conflictos Armados Internacionales, lo cual dio lugar a los Cuatro Convenios de Ginebra de 1949.

Estos documentos se convirtieron en la piedra angular del DIH. Nacieron del querer de los gobiernos, de las Sociedades Nacionales y de la Cruz Roja. En este proceso, el Comité reunió la documentación preliminar para poner en conocimiento los puntos que debían ser modificados, completados o confirmados, y con la colaboración de expertos internacionales redactaron los borradores de los cuatro Convenios para someterlos a votación en la Conferencia Internacional de la Cruz Roja (en la cual participan gobiernos y las Sociedades Nacionales). En el ańo 1948 se terminaron los documentos que legítimamente fueron aceptados y legalmente concebidos por la comunidad internacional y que hoy en día regulan las guerras o los conflictos armados internacionales (Los Convenios de Ginebra de 1949, nota preliminar).

Hasta la Segunda Guerra Mundial, la tipología de los conflictos era de carácter internacional, por lo que las normas contempladas en los Convenios mencionados se convierten en la norma que determina, en la guerra, la legalidad del comportamiento de los combatientes.

Desafortunadamente, estas normas se quedaron cortas cuando la tipología de los conflictos armados cambió y se generalizó el Conflicto Armado No Internacional (Cani). Esto trajo consigo un gran problema, pues los Estados estaban dispuestos a someter a sus ejércitos a unas normas que regulaban la guerra internacional, pero dichas normas no tienen la legitimidad para ser aplicadas en el interior de cada Estado en caso de conflicto armado no internacional, salvo el Artículo 3, común a los Convenios.

Por lo anterior, los Estados debían someter sus normas y los comportamientos de los combatientes a normas supraestatales, sean estos combatientes estatales o no estatales. De manera que se convocó a una nueva Conferencia Internacional que dio origen a los dos Protocolos Adicionales de 1977, cuyo II Protocolo rige los Conflictos Armados No Internacionales.

Los tratados de DIH contemplan unos mínimos para mitigar los efectos terribles de los enfrentamientos armados, lo cual beneficia a quienes participan directamente de las hostilidades y, por ese simple hecho, debería ser aceptado y respetado por todas las partes en conflicto, indistintamente si son fuerzas legítimas del Estado o disidentes. 
Además de lo anterior, la Corte Constitucional colombiana determinó en la Sentencia C95/225- que las normas del Derecho Internacional Humanitario son parte integrante del ius cogens, y al tenor de la Convención de Viena de 1969, se trata de una norma aceptada y reconocida por todos los Estados que no admite acuerdo en contrario y solo puede ser modificada por una norma ulterior del Derecho Internacional. Explica la Corte que por ello las normas que constituyen el DIH son de obligatorio cumplimiento para los Estados y las partes involucradas en las hostilidades, por cuanto las normas que constituyen el DIH son de carácter consuetudinario, toda vez que el DIH es una recopilación de viejas prácticas consuetudinarias.

Continúa la Alta Corte explicando que el DIH es obligatorio para quienes participan en los conflictos armados, por lo cual los grupos armados no estatales no se pueden escudar en su no ratificación para incumplir los mínimos de humanidad en la guerra que deriva de la universal aceptación de su normatividad por los pueblos civilizados (Corte Constitucional, 1995).

En todo caso, y a pesar de que los Estados no tengan suscrito y ratificado el II Protocolo Adicional de 1977, la Cláusula Martens obliga a respetar unos mínimos en aras de proteger a quienes no combaten y conservar los principios de la humanidad. Dicha cláusula, legítimamente constituida, es la base legal para determinar el comportamiento de los combatientes en conflictos armados no internacionales, contemplada en el Artículo 3 común a los Convenios de 1949 y por lo tanto universalmente aceptada (Sassòli \& Bouvier, 2006). No obstante, la constante transgresión a las normas que conforman el DIH en los conflictos armados no internacionales ponen en entredicho su eficacia.

Con el transcurso de los años, el CICR no ha cesado sus esfuerzos para que se reconozcan, en el derecho de gentes, los mínimos necesarios para proteger el ser humano en caso de guerra. Con tal fin, impulsa convenios humanitarios y promueve alrededor del mundo la protección debida a las normas existentes. No obstante, la realidad supera aún más al derecho y se observa que los convenios actuales se quedan cortos ante los desafíos que traen las nuevas tipologías de conflicto y el avance de la tecnología militar.

Asimismo, la Resolución 2444 de 1968 de la Asamblea General de las Naciones Unidas, así como las dos conferencias realizadas por el Movimiento Internacional de la Cruz Roja y Naciones Unidas buscaban fomentar prácticas humanitarias en lo referente a la protección de los civiles de los ataques indiscriminados en los conflictos armados, especialmente los no internacionales. Como resultado de dichas conferencias, la Asamblea General se apropió de sus principios de protección y afirmó que los combatientes tienen límites en su actuación y, por lo tanto, no pueden causar daños innecesarios contra sus enemigos, así como en todo momento deben distinguir entre civiles y combatientes. Igualmente, solicitó a los Estados miembros de Naciones Unidas que no hayan suscrito las Convenciones de la Haya de 1899 y 1907, el Protocolo de Ginebra de 1925 y los Convenios de Ginebra de 1949 que los suscribieran y ratificaran (Asamblea General de Naciones Unidas, 1968).

Las conferencias mencionadas anteriormente aceleraron un movimiento que propendía por hacer confluir las diferentes corrientes que regulaban la protección de los no combatientes y los límites en la guerra, es decir, la unión del derecho de La Haya referente a la conducción de hostilidades, el derecho de Ginebra relativo a la protección de las víctimas de los conflictos y, finalmente, los tratados suscritos en Nueva York en lo referente a los Derechos Humanos (Doménech, 2007). 
El I Protocolo adicional de 1977, de aplicación en los Conflictos Armados Internacionales (CAI), es un compendio de normas que busca resaltar la importancia de los principios básicos del Derecho Internacional Humanitario. Por esta razón no podía quedarse sin una sección destinada a los límites referidos a los métodos y medios de guerra, de manera que dedica los artículos 35 a 42 a la aplicación de los principios del DIH en la conducción de las hostilidades. Asimismo, en este documento no solo se prohíben las armas que causan daños permanentes al medio ambiente y que impliquen medios pérfidos, sino que también trae una prohibición para el desarrollo de nuevo armamento.

Teniendo en cuenta que en su Artículo 36 limita los nuevos medios, es importante comprender qué significan las palabras medios y métodos en la guerra. Estas hacen referencia a las armas y prácticas que los combatientes emplean en la guerra con la finalidad de debilitar y doblegar al enemigo, entendiendo medios como las armas y sistemas armados, y los métodos como las estrategias y tácticas de guerra.

Asimismo, el Artículo 36 del I Protocolo adicional a los Convenios de Ginebra determina el marco jurídico para hacer el examen de las nuevas armas. Afirma que es obligación de las partes establecer si el empleo de las armas puede ser o no aplicable sin violar las normas del DIH. No obstante, no se estipuló en el Protocolo cómo se debe hacer el procedimiento ni quién es el responsable de realizarlo en los Estados en conflicto.

Precisamente, cabe anotar que el ánimo de este artículo es prevenir el desarrollo de armas que atentan contra la integridad del enemigo de manera innecesaria y que por lo mismo violan los principios del DIH (Comité Internacional de la Cruz Roja [CICR], 2006).

Ahora bien, para cumplir con el objetivo del Artículo 36 hace falta que los Estados revisen constantemente su producción y adquisición de nuevo armamento, y que dicha revisión, preferiblemente, se realice tanto por las altas partes que tienen suscrito y ratificado el I Protocolo Adicional, como por aquellos Estados que no lo han hecho. Esto se debe a que la aplicación de dicho armamento puede o no afectar a combatientes y a no combatientes de Estados no partes del protocolo. No obstante, son pocos los Estados que han instaurado mecanismos de verificación de sus armas, aspecto por el cual se pueden destacar Bélgica, Australia, Estados Unidos, Holanda, Noruega y Suecia (CICR, 2006, p. 5).

Ahora bien, para prohibir el arma es necesario que esta haya sido concebida de tal forma que viola uno de los dos grandes principios consagrados por el I Protocolo Adicional: que el arma cause sufrimiento innecesario o males superfluos (art. 35-2) y que cause actos indiscriminados (art. 54 b-4).

En este sentido, el desafío consiste en que aún no se han desarrollado en su totalidad los armamentos autónomos y por lo tanto no hay norma ni regulación que a nivel mundial prohíba ciertos comportamientos de las armas autónomas. Las normas existentes han sido concebidas para ser respetadas por los combatientes, no por sus armas. El gran problema del derecho es que no tiene una capacidad prospectiva para ser promulgado, de manera que los avances tecnológicos siempre representan retos para el DIH.

Finalmente, en el corazón de las Naciones Unidas se están discutiendo los retos jurídicos que los avances tecnológicos representan para los Estados. En aras de ello, vale la pena resaltar las palabras del delegado de Estados Unidos en el Informal Meeting de abril de 2015: 
we expect this week's discussion to deepen our understanding of the complex issues surrounding LAWS as our program of work will allow us to more fully explore this topic. That said, we believe that it is important to focus on increasing our understanding versus trying to decide possible outcomes. It remains our view that it is premature to try and determine where these discussions might or should lead [...]. (U.S. Delegation Opening Statement as Delivered by Michael W. Meier Geneva, 2015)

Como lo expresó la Delegación estadounidense, tal vez sea prematuro hablar de leyes si no hay comprensión del funcionamiento y alcance de la tecnología emergente.

\section{Conclusiones}

El desarrollo científico y tecnológico de la inteligencia artificial ha conllevado la generación de un nuevo armamento que llama a los Estados productores a preguntarse por las implicaciones éticas y legales de emplearlo.

Es así como preguntas tan complejas como, por ejemplo, las formuladas sobre la responsabilidad de las actuaciones de un robot en el campo de batalla, implican, en primera medida, la revisión del propio armamento en los términos que estipula el Artículo 36 del I Protocolo Adicional de 1977. Asimismo, se hace indispensable contrarrestar los efectos que estas armas, eventualmente, pueden representar en las hostilidades cuando dichas actuaciones violen las disposiciones del DIH.

El artículo 36 del I Protocolo Adicional se puede quedar corto, toda vez que por más revisión del armamento que realicen las partes, se trata de una autoevaluación y no existen más garantías que las normas escritas y los principios del DIH que buscan limitar el uso de armamento indiscriminado y que causa sufrimiento innecesario.

No obstante, y tal como lo afirma la agencia estadounidense Defense Advanced Research Projects Agency (Darpa, 2015), el armamento debe ser producido siguiendo las normas existentes. El problema está en que países como los Estados Unidos no forman parte de los Protocolos Adicionales y otros varios tratados que limitan los medios y modos de hacer la guerra, por lo cual las únicas normas existentes para estos son las normas consuetudinarias y los principios del DIH que se caracterizan por su universalidad.

En segunda medida, si se trata de limitar el armamento autónomo, es indispensable que tanto ingenieros, militares como abogados hablen el mismo lenguaje. Para ello es necesario comprender cómo funciona y qué alcance tienen estos sistemas. En aras de ello, los Meeting informales de las Naciones Unidas realizados en Ginebra desde el 2012 constituyen el primer acercamiento y comprensión de la tecnología emergente.

Finalmente, para lograr los objetivos de limitar los métodos y los medios para hacer la guerra y de proteger a los que no combaten, todos los Estados deberían estar sometidos a los Protocolos Adicionales, entre los cuales se destaca la autoevaluación regular del nuevo armamento y su prohibición en cuanto que causaren daños superfluos y sufrimientos innecesarios. En el mismo sentido, los Estados deberían cumplir con lo establecido por los principios generales del DIH, universales y de obligatorio respeto, lo cual permitiría agilidad y flexibilidad en el derecho internacional. 


\section{Referencias}

1. Anderson, K., \& Waxman, M. (2013). Law and Ethics for Autonomous Weapon Systems. Why a Ban Won't Work and How the Laws of War Can [documento en línea]. Stanford: Jean Perkins Task Force on National Security and Law. Recuperado de: http://www.hoover.org/sites/default/files/ uploads/documents/Anderson-Waxman_LawAndEthics_ r2_FINAL.pdf

2. Asamblea General de Naciones Unidas. (1968). Resolución 2444 (XXIII) Respeto de los Derechos Humanos en los Conflictos Armados. 1748 plenaria [documento en línea]. Recuperado de http://www.un.org/es/comun/docs/?symbol=A/RES/2444(XXIII)

3. Bugnion, F. (2001). El derecho de Ginebra y el derecho de la Haya. Revista Internacional de la Cruz Roja. 844, 901-922.

4. Carrillo Salcedo, J. A. (2001). Soberanía de los Estados y Derechos Humanos en el Derecho Internacional Contemporá-neo (2.a ed.). Madrid: Tecnos.

5. Comité Internacional de la Cruz Roja (CICR). (2006). Guía para el examen jurídico de las armas, los medios y los métodos de guerra nuevos. Medidas para aplicar el Artículo 36 del Protocolo Adicional de 1977. Ginebra.

6. Comité Internacional de la Cruz Roja. (1986). Los Cuatro Convenios de Ginebra de 1949. Ginebra: Comité Internacional de la Cruz Roja.

7. Convención II de La Haya de 1899 relativa a las Leyes y usos de la guerra terrestre y reglamento anexo [documento en línea]. Recuperado de http://www.cruzroja.es/dih/pdf/II_convenio_de_la_haya_de_1899_relativa_a_leyes_usos_ouerra_terrestre_y_reglamento_anexo.pdf

8. Corte Constitucional de Colombia. (1995). Sentencia C-225/95. MP: Alejandro Martinez.

9. Defense Advanced Research Projects Agency (Darpa). (2015). Breakthrough Technologies for National Security [documento en línea]. Recuperado de http://www.darpa. mil/attachments/DARPA2015

10. Doménech Omedas, J. L. (2007). Limitaciones al empleo del medio y métodos de combate: armas convencionales excesivamente dańinas o efectos indiscriminados. Residuos explosivos y proliferación de armas ligeras. En Rodríguez-Villasante y José Luis Prieto (Eds.), Humanitario [Tirant, monografías 225] (pp. 311-353). Valencia: Cruz Roja Espańola.

11. Dunant, H. (2010). Recuerd de Solferino. Barcelona : Creu Roja Catalunya.

12. Ejército Nacional, Fuerzas Militares de Colombia. (2010). Reglamento de operaciones y maniobras de combate irregular. Reglamento EJC 3-10-1. Bogotá: Publicaciones Ejército.
13. Herrera, D. A. (junio, 2008). Ius gentium ¿Derecho natural o positivo? Trabajo presentado en De Iustitia Et Iure En El Siglo De Oro. Terceras Jornadas Internacionales: Ley, Guerra y Paz en Francisco Suárez. Pontificia Universidad Católica Argentina, Santa María de los Buenos Aires. Recuperado de https://fccuniversitario.files.wordpress.com/2012/06/herrera-ius-gentium-derecho-natural-o-derecho-positivo.pdf

14. Horowitz, M. C., \& Scharre, P. (2015). Meaningful Human Control in Weapon Systems: A Primer [Working Paper]. Center for a New American Security.

15. International Committee of the Red Cross (ICRC). (2012). New technologies and warfare. International Review of the Red Cross, 886, 457-466.

16. I y II Protocolo Adicional de 1977. Comité Internacional de la Cruz Roja. Ginebra.

17. Kalshoven, F. \& Zegveld, L. (2005). Restricción en la conducción de la guerra: Introducción al Derecho Internacional Humanitario. Ginebra: Comité Internacional de la Cruz Roja.

18. Khun, T. (1970). The Structure of Scientific Revolutions (Vol. II, num. 2). University of Chicago.

19. Mejía Azuero, J. C. \& Chaíb de Mares, K. (2012). Derecho de la Guerra. Bogotá: Equión Energía Limited y Fundación Universitaria Los Libertadores.

20. Pictet, J. (1998). Desarrollo y principios del Derecho Internacional Humanitario. Ginebra: TM Editores, Instituto Henry Dunant, CICR Ginebra.

21. Plazas Vega, M. (2006). La filosofía kantiana de la moral, el derecho y la políitica: de la "razón pura teórica" a la "razón pura práctica”. En Oscar Alberto Delgado Sánchez (Comp.), Ideas políticas, filosofía y derecho: El maestro (pp. 228259). Bogotá: Universidad del Rosario.

22. Rodriguez-Villasante \& Prieto, J. L. (2007). Derecho Internacional Humanitario [Tirant, monografías 225]. Valencia: Cruz Roja Española.

23. Salmón, E. (2004). Introducción al Derecho Internacional Humanitario [archivo de libro en línea]. Recuperado de http://www.corteidh.or.cr/tablas/r25212.pdf

24. Sassòli, M., \& Bouvier, A. (2006). How Does Law Protect in War? Geneva: International Committee of the Red Cross.

25. Segovia, J. F. (2014). Las cuestiones de Locke sobre la ley natural. Examen crítico de sus principales argumentos. Derecho Público Iberoamericano, 4, 167-209. Recuperado de file:///Users/marthahurtado/Downloads/Dialnet-LasCu estionesDeLockeSobreLaLeyNatural-4790074.pdf 
26. Swinarski, Ch. (2015/2003). Del terrorismo en el derecho internacional público. En Juan Ramón Martínez Vargas (Ed.), Derecho Internacional y Terrorismo (pp. 44-45). Universidad el Rosario.

27. The Convention on Certain Conventional Weapons (CCM). (2014). Meeting of Experts on Lethal Autonomous Weapons Systems [página web]. Recuperado de http://www.unog.ch/_80256ee600585943.nsf/(httpPages)/a038dea1da906f9dc1257dd90042e261?OpenDocumenterExpandSection=1\#_Section 1

28. The Convention on Certain Conventional Weapons (CCM). (2015). Meeting of Experts on Lethal Autonomous Weapons Systems [página web]. Recuperado de http:// www.unog.ch/80256EE600585943/(httpPages)/6CE049BE22EC75A2C1257C8D00513E26?OpenDocument

29. The Red Cross and the Red Crescent. (2011). 31st International Conference, International Humanitarian Law and the Challenges of Contemporary Armed Conflicts. Geneve.
30. U.S. Delegation Opening Statement as Delivered by Michael W. Meier Geneva. (April, 2015). The Convention on Certain Conventional Weapons (CCW) Informal Meeting of Experts on Lethal Autonomous Weapons Systems [documento en línea]. Recuperado de http://www.unog. ch/80256EDD006B8954/(httpAssets)/8B33A1CDBE80EC60C1257E2800275E56/\$file/2015_LAWS_MX_USA+bis. pdf

31. United States, Department of Defense. (2012). Autonomy in Weapon Systems, 3000.09 [documento en línea]. Recuperado de: www.dtic.mil/whs/directives/corres/pdf/300009p.pdf

32. Verdoss, A. (1967). Derecho Internacional Público. Madrid: Biblioteca Jurídica Aguilar.

33. Zenko, M. (2013). Reforming U.S. Drone Strike Policies. Council on Foreign Relations. January, Recuperado de http:// www.cfr.org/wars-and-warfare/reforming-us-drone-strike-policies/p29736. 